\title{
Jet evolution in a dense medium: event-by-event fluctuations and multi-particle correlations
}

\section{Miguel Ángel Escobedo*}

Department of Physics, P.O. Box 35, 40014 University of Jyväskylä, Finland

Institut de Physique Théorique, Université Paris Saclay, CNRS, CEA, F-91191, Gif-sur-Yvette,

France

E-mail: miguel.a.escobedo-espinosa@jyu.fi

\section{Edmond lancu}

Institut de Physique Théorique, Université Paris Saclay, CNRS, CEA, F-91191, Gif-sur-Yvette,

France

E-mail: edmond.ianculcea.fr

\begin{abstract}
We study the gluon distribution produced via successive medium-induced branchings by an energetic jet propagating through a weakly-coupled quark-gluon plasma. We show that under suitable approximations, the jet evolution is a Markovian stochastic process, which is exactly solvable. For this process, we construct exact analytic solutions for all the n-point correlation functions describing the gluon distribution in the space of energy [1,2]. Using these results, we study the event-by-event distribution of the energy lost by the jet at large angles and of the multiplicities of the soft particles which carry this energy. We find that the event-by-event fluctuations are huge: the standard deviation in the energy loss is parametrically as large as its mean value [1]. This has important consequences for the phenomenology of di-jet asymmetry in $\mathrm{Pb}+\mathrm{Pb}$ collisions at the LHC: it implies that the fluctuations in the branching process can contribute to the measured asymmetry on an equal footing with the geometry of the di-jet event (i.e. as the difference between the in-medium path lengths of the two jets). We compute the higher moments of the multiplicity distribution and identify a remarkable regularity known as Koba-Nielsen-Olesen (KNO) scaling [2]. These predictions could be tested via event-by-event measurements of the di-jet asymmetry.
\end{abstract}

The European Physical Society Conference on High Energy Physics

5-12 July, 2017

Venice

${ }^{*}$ Speaker. 


\section{Introduction}

One of the observables in which the formation of a collective medium in heavy-ion collisions manifests in a very clear way is the dijet asymmetry, the energy difference between two approximately back-to-back jets $[3,4]$. The usual interpretation of this observation is the following:

- The two energetic jets are initially created in a hard process, due to momentum conservation the two jets will have back-to-back directions and approximately the same energy.

- For simplicity we consider central collisions in which the interaction plane has rotational symmetry. The point of the collision region in which the hard process takes place is not always the center, a deviation from this point will have as a consequence that the effective size of the medium seen by each jet will be different.

- The two jets will lose energy inside of the medium, however the amount of energy loss will depend on the size of the medium that they traverse.

However, this might not be the whole story. In the previous discussion we were assuming that the energy loss is always the same at fixed medium size, in other words, we were neglecting fluctuations. The question is then, how big are these fluctuations? This is one of the main problems we are going to address in this proceedings and the answer we are going to find is that the typical deviation in the energy loss is of the order of the average value and therefore fluctuations can not be neglected. We are going to arrive to this conclusion by performing an analytical computation based on the results obtained in [5, 6]. A similar result was obtained recently by a Monte Carlo computation in [7]. Another issue we want to discuss in these proceedings is what are the eventby-event properties of gluons produced by the energy loss mechanism.

\section{Jet quenching formalism}

We are going to perform the computation using the BDMPS-Z theory $[8,9]$. In this formalism all the information that we need from the medium is encoded in its length $L$ and a parameter called $\hat{q}$. There are two time-scales that have a very important role in this problem. One is the emitted gluon formation time $\tau_{f} \sim \sqrt{(2 \omega) / \hat{q}}$. Another important time-scale is the branching time. In the BDMPS-Z theory the probability to emit a gluon during a small time $\Delta t$ is

$$
P(\omega, \Delta t) \propto \frac{N_{c} \alpha_{s}}{\pi} \sqrt{\frac{\hat{q}}{\omega}} \Delta t
$$

the branching time $\tau_{b r}$ is the period after which we are almost sure that a gluon with a given energy will be emitted, looking at the previous equation we can see that $\tau_{b r}(\omega)=\frac{\pi}{N_{c} \alpha_{s}} \tau_{f}(\omega)$, this shows that in perturbation theory the formation time is much smaller than the branching time and therefore, at first approximation, the branching process can be thought as an almost classical process in which gluons are formed instantaneously.

The branching time allows to divide the gluons in two different types. On one hand we have soft gluons that have an energy such that $\tau_{b r} \ll L$ therefore they will be emitted abundantly. On the other hand we have the class of the hardest gluons which are likely to be emitted. They are 
those with $\tau_{b r} \sim L$, this implies that they will have an energy of order $\omega_{b r} \sim \alpha_{s}^{2} \hat{q} L^{2}$. Their emission by the leading particle will dominate the energy loss. However, this gluons with energy $\omega_{b r}$ will subsequently branch and at the end of the day what will be found is a lot of soft gluons emitted at large angles. The equations and the consequences of the multiple branching obtained with the previous assumptions were discussed in [5, 6], there it was observed the importance of the so-called democratic branching, the process in which a parton branches in a way such that the resulting partons have a similar energy. This will be a rare event for the leading particle because their energy is much bigger than $\omega_{b r}$, however for the gluons emitted by the leading particle, that will typically have an energy of the order of $\omega_{b r}$ or smaller, this will be a very common process and a very efficient way to transfer energy into low energy gluons emitted at large angles.

\section{The gluon spectrum and the average energy loss}

The main focus of this section is going to be the gluon spectrum that we define as $D(x, t)=$ $x\left\langle\sum_{i} \delta\left(x_{i}-x\right)\right\rangle$, where $x$ is the energy fraction carried by the gluon. This quantity evolves with time following the equation [10]

$$
\frac{\partial}{\partial \tau} D(x, \tau)=\int \mathrm{d} z \mathscr{K}(z)\left[\sqrt{\frac{z}{x}} D\left(\frac{x}{z}, \tau\right)-\frac{z}{\sqrt{x}} D(x, \tau)\right]
$$

where $\tau=\frac{\alpha_{s} N_{c}}{\pi} \sqrt{\frac{\hat{q}}{E}} t=\frac{t}{\tau_{b r}(E)}$. $E$ in this case is the energy of the leading particle. In [6] eq. (3.1) was solved with the approximate kernel $\mathscr{K}_{0}(z)=\frac{1}{[z(1-z)]^{3 / 2}}$ and the initial condition $D(x, 0)=\delta(1-x)$

$$
D(x, \tau)=\frac{\tau}{\sqrt{x}(1-x)^{3 / 2}} \exp \left\{-\frac{\pi \tau^{2}}{1-x}\right\} .
$$

The previous formula implies that the energy decreases with time, in fact

$$
\langle X(\tau)\rangle=\int_{0}^{1} d x D(x, \tau)=e^{-\pi \tau^{2}}
$$

so the energy loss is $\mathscr{E}(\tau)=E\left(1-e^{-\pi \tau^{2}}\right)$.

\section{The 2-point function and the fluctuations of the energy loss}

In order to quantify the importance of the energy loss fluctuations we will compute the variance $\sigma_{\mathscr{E}}^{2}=E^{2}\left(\left\langle X^{2}\right\rangle-\langle X\rangle^{2}\right)$. We already computed the value of $\langle X(\tau)\rangle$. In order to compute $\left\langle X^{2}(\tau)\right\rangle$ apart from $D(x, \tau)$ we also need the 2-point function defined as

$$
D^{(2)}\left(x, x^{\prime}, t\right)=x x^{\prime}\left\langle\sum_{i \neq j} \delta\left(x_{i}-x\right) \delta\left(x_{j}-x^{\prime}\right)\right\rangle,
$$

which gives information about the pairs of partons with different energy found inside the jet. Knowing this $\left\langle X^{2}\right\rangle$ is determined as

$$
\left\langle X^{2}(t)\right\rangle=\int_{0}^{1} d x x D(x, t)+\int_{0}^{1} d x \int_{0}^{1} d x^{\prime} D^{(2)}\left(x, x^{\prime}, t\right) .
$$


$D^{(2)}$ fulfills an evolution equation similar to the one in eq. (3.1), under the same approximations leading to eq. (3.2) the evolution equation of $D^{(2)}$ has the solution

$$
D^{(2)}\left(x, x^{\prime}, \tau\right)=\frac{1}{2 \pi} \frac{1}{\sqrt{x x^{\prime}\left(1-x-x^{\prime}\right)}}\left[\mathrm{e}^{-\frac{\pi \tau^{2}}{1-x-x^{\prime}}}-\mathrm{e}^{-\frac{4 \pi \tau^{2}}{1-x-x^{\prime}}}\right] .
$$

With this result we can already compute $\sigma_{\mathscr{E}}^{2}$. In the limit $\tau \ll 1$, which is the one interesting for LHC physics

$$
\sigma_{\mathscr{E}}^{2}(\tau)=E^{2}\left(\frac{1}{3} \pi^{2} \tau^{4}-\frac{11}{15} \pi^{3} \tau^{6}\right)+\mathscr{O}\left(E^{2} \tau^{8}\right)
$$

this result means that the typical deviation will go like $E \tau^{2} \sim \omega_{b r}$. This means that both the average and the typical deviation are of the same order of magnitude and that both are of the size of $\omega_{b r}$.

Let us now discuss the phenomenological consequences of this result. Suppose that in a heavyion collision two jets are created with the same energy in the center of the collision so that both jets see the same medium path length. Our result shows that even in this situation the difference between the energy loss of the two jets might be of the same order of magnitude as the energy loss itself.

\section{The n-point functions and KNO scaling}

In order to compute the average energy loss $\langle\mathscr{E}\rangle$ and the average number of particles inside the jet $\langle N\rangle$ we need to know the gluon spectrum $D$. If we want to compute $\left\langle\mathscr{E}^{2}\right\rangle$ and $\left\langle N^{2}\right\rangle$ we also need to know $D^{(2)}$. If we want to have more detailed information on the energy loss and the distribution of particles we need to compute higher order n-point functions $D^{(n)}$. They fulfill an evolution equation similar to the one of $D^{(2)}$ and they can be analytically solved using the same approximations [2]. We can use this result to compute the ratio of the moments of the distribution of soft particles inside the jet with respect to the mean number of these particles

$$
C_{p}=\frac{\left\langle N^{p}\right\rangle}{\langle N\rangle^{p}}=\frac{(p+1) !}{2^{p}},
$$

which is a constant that only depends on $p$. This property is called KNO scaling [11] and appears in several processes in heavy-ion as well as in collider physics. In fact, eq. (5.1) corresponds to a negative binomial distribution with parameter $k=2$. Similar properties were also found in the vacuum [12], there it was seen that KNO scaling is also fulfilled and that the distribution of emitted gluons was approximately described by a negative binomial distribution but this time with $k=3$. In conclusion we can see that the distribution of gluons produced by a jet, either in a medium or in the vacuum, can be approximately described by a negative binomial distribution and therefore they approximately fulfill KNO scaling. The difference is that in a medium fluctuations and correlations are much more important.

\section{Conclusions}

In this proceedings we have reviewed the computation of the fluctuations of the energy loss. We have seen that they are large, of the order of the average value. This means that they can not 
be neglected when interpreting experimental results. This is particularly important for the dijet asymmetry, our result shows that such an asymmetry can be generated even if the medium path length that each jet traverses is the same. This is in contradiction with the usual picture.

We have also shown that the gluons emitted during the process in which the jet loses energy fulfill KNO scaling and can be approximately described by a negative binomial distribution. Remarkably this is similar to what is found in the vacuum where the physics is very different. Comparing the two cases we see that in the medium correlations and fluctuations are much bigger.

\section{Acknowledgments}

The work of M.A.E. has been supported, during the preparation of the talk and the proceedings, in part by the European Research Council under the Advanced Investigator Grant ERC-AD-267258 and in part by the Academy of Finland, project 303756.

\section{References}

[1] M. A. Escobedo and E. Iancu, Event-by-event fluctuations in the medium-induced jet evolution, JHEP 05 (2016) 008, [1601.03629].

[2] M. A. Escobedo and E. Iancu, Multi-particle correlations and KNO scaling in the medium-induced jet evolution, JHEP 12 (2016) 104, [1609.06104].

[3] ATLAS collaboration, G. Aad et al., Observation of a Centrality-Dependent Dijet Asymmetry in Lead-Lead Collisions at $\sqrt{s_{N N}}=2.77 \mathrm{TeV}$ with the ATLAS Detector at the LHC, Phys. Rev. Lett. 105 (2010) 252303, [1011.6182].

[4] CMS collaboration, V. Khachatryan et al., Correlations between jets and charged particles in PbPb and pp collisions at $\sqrt{s_{\mathrm{NN}}}=2.76 \mathrm{TeV}$, JHEP 02 (2016) 156, [1601.00079].

[5] J.-P. Blaizot, F. Dominguez, E. Iancu and Y. Mehtar-Tani, Probabilistic picture for medium-induced jet evolution, JHEP 06 (2014) 075, [1311 . 5823].

[6] J.-P. Blaizot, E. Iancu and Y. Mehtar-Tani, Medium-induced QCD cascade: democratic branching and wave turbulence, Phys. Rev. Lett. 111 (2013) 052001, [1301.6102].

[7] J. G. Milhano and K. C. Zapp, Origins of the di-jet asymmetry in heavy ion collisions, Eur. Phys. J. C76 (2016) 288, [1512.08107].

[8] R. Baier, Y. L. Dokshitzer, A. H. Mueller, S. Peigne and D. Schiff, Radiative energy loss of high-energy quarks and gluons in a finite volume quark - gluon plasma, Nucl. Phys. B483 (1997) 291âĂŞ320, [hep-ph/9607355].

[9] B. G. Zakharov, Fully quantum treatment of the Landau-Pomeranchuk-Migdal effect in QED and QCD, JETP Lett. 63 (1996) 952âĂŞ957, [hep-ph / 9607440 ].

[10] R. Baier, A. H. Mueller, D. Schiff and D. T. Son, 'Bottom up' thermalization in heavy ion collisions, Phys. Lett. B502 (2001) 51âĂŞ58, [hep-ph/ 0009237 ].

[11] Z. Koba, H. B. Nielsen and P. Olesen, Scaling of multiplicity distributions in high-energy hadron collisions, Nucl. Phys. B40 (1972) 317âĂŞ334.

[12] Y. L. Dokshitzer, V. A. Khoze, A. H. Mueller and S. I. Troian, Basics of perturbative QCD. 1991. 